\title{
EDITORIAL
}

\section{Charity is not enough}

Three years on, little or no progress has been made towards achieving the Millennium

Development Goals. The actions of charitable foundations and agencies should work in

concert with, rather than instead of, the actions of governments.

When did you last read a feature on malaria, a news story on HIV or a research paper on TB? These infectious diseases are all major killers and constitute a huge drain on public health resources in every region of the world. The HIV/AIDS pandemic, the re-emergence of tuberculosis and the continuing struggle against malaria are deservedly attracting considerable resources from both the public and private sectors, as the recent announcement from the Bill \& Melinda Gates Foundation (BMGF) illustrates and, as the BMGF announcement also showed, they receive extensive attention from the media.

The fact remains, however, that the leading cause of death in children under five is not HIV/AIDS, malaria or TB but acute respiratory infections (ARIs) — often in the form of Streptococcus pneumoniae or Haemophilus influenzae pneumonia - which kill more than two million children each year. Another two million children under the age of five succumb to diarrhoeal diseases. And the measles virus still infects more than 40 million children worldwide and is the biggest vaccine-preventable killer of children, claiming the lives of more than 800,000 under-fives annually. This year, almost 11 million children will die before their fifth birthday, almost all in poor nations. Although HIV/AIDS and TB do affect child mortality rates, fundamentally it is the same infectious diseases that were killing children 50 years ago that still cause most childhood deaths today.

The real tragedy behind these appalling statistics is that most of these deaths are preventable. In most cases, pneumonia can be effectively treated with low-cost oral antibiotics. According to WHO estimates, the correct management of ARIs could save the lives of 1 million children per year. Diarrhoea is easily treated with straightforward oral-rehydration therapy; appropriate management of diarrhoeal diseases could save an estimated 1.8 million children each year. And effective use of the measles vaccine could save an estimated 700,000 lives per year.

These major public health issues have in general received little media attention, compared with HIV/AIDS or malaria, and yet the signs are that the situation is getting worse, not better. In a recent UNICEF study published by the Townsend Centre for International Poverty Research at the University of Bristol and the London School of Economics, which assessed more than 1 million children from 46 developing countries, it was reported that some $15 \%$ of subjects (more than 250 million children) had never been immunized or were suffering from chronic diarrhoea that had never been treated, and more than 1 billion children are living in severely deprived conditions

In September 2000, the United Nations adopted the Millennium Declaration, which was translated into eight specific goals known as the Millennium Development Goals, with a target date for achievement of 2015. The goals include a $50 \%$ reduction in poverty and extreme hunger, halting and reversing the spread of HIV/AIDS, malaria and other infectious diseases, and reducing child mortality by two-thirds. So, what has happened? Was the Millennium Declaration an empty gesture? The difficulties of translating objectives into real progress on a global scale are well known and understandable, particularly for worldwide bodies like the UN. But the necessary tools, treatments and technologies required to intervene effectively in these crises are all available right now. What is lacking at the highest level is the political will and commitment to ensure that these resources are made available to the people who need them most.

We applaud the efforts of private foundations such as the BMGF. In a recent announcement concerning the Grand Challenges in Global Health initiative, the Executive Director of the global health programme at the BMGF, Dr Richard Klausner, remarked, 'It is high time that the world's scientific community, which has contributed so much to the medical progress achieved in the last century, turns its creative attention to solving the enormous health problems of the developing world.' We couldn't agree more, and as microbiologists — whether working in clinical, industrial or fundamental areas of research this could be an area in which you can make a difference. But the actions of charitable foundations are not enough. It is time for governments to live up to their commitments. 